\title{
Distributed Visual-Vestibular Processing in the Cerebral Cortex of Man and Macaque
}

\author{
Andrew T. Smith ${ }^{1, *}$, Mark W. Greenlee ${ }^{2}$, Gregory C. DeAngelis ${ }^{3}$ and \\ Dora E. Angelaki ${ }^{4}$ \\ ${ }^{1}$ Department of Psychology, Royal Holloway, University of London, Egham TW20 0EX, UK \\ ${ }^{2}$ Institute of Experimental Psychology, University of Regensburg, 93053 Regensburg, \\ Germany \\ ${ }^{3}$ Department of Brain and Cognitive Sciences, University of Rochester, Rochester, \\ New York 14627, USA \\ ${ }^{4}$ Department of Neuroscience, Baylor College of Medicine, Houston, Texas 77030, USA
}

Received 22 March 2017; accepted 28 March 2017

\begin{abstract}
Recent advances in understanding the neurobiological underpinnings of visual-vestibular interactions underlying self-motion perception are reviewed with an emphasis on comparisons between the macaque and human brains. In both species, several distinct cortical regions have been identified that are active during both visual and vestibular stimulation and in some of these there is clear evidence for sensory integration. Several possible cross-species homologies between cortical regions are identified. A key feature of cortical organization is that the same information is apparently represented in multiple, anatomically diverse cortical regions, suggesting that information about self-motion is used for different purposes in different brain regions.
\end{abstract}

Keywords

Visual, vestibular, MST, VIP, PIVC, VPS, PIC, CSv

\section{Introduction}

In daily life, both humans and macaques are constantly on the move. Movement can only be effective if we continuously monitor our location in the external world and our trajectory through it. The principal sensory cues that

\footnotetext{
* To whom correspondence should be addressed. E-mail: a.t.smith@rhul.ac.uk 
enable us to perceive self-motion are visual and vestibular. In the case of vision, multiple cues are available but we make heavy use of what Gibson (1950) termed optic flow: the orderly, two-dimensional pattern of image motion cast on the retina by our surroundings as we move. Optic flow provides a rich source of information from which can be extracted our instantaneous direction of heading during locomotion (Warren and Hannon, 1988). If additional information about object distance is available for scaling, as it usually is, optic flow can in principle be used to establish the speed at which we are moving. However, the necessary computations are not trivial. During locomotion, we usually want to know about the heading of the whole body but optic flow specifies self-motion only in the coordinates of the eyes. The eyes move relative to the head and the head moves relative to the body. Only if these movements are known and factored in can whole-body heading be established (see for example Lappe et al., 1999). Such information is available but the transformation is complex and may be error-prone. Another limit of visual cues to self-motion is that the retinal image contains not only flow generated by self-motion but also motion due to the movement of the objects around us. Only if these different sources of image motion can be parsed is accurate perception of self-motion possible (Royden and Hildreth, 1996; Warren and Rushton, 2009).

The vestibular system provides complimentary information. It offers several key advantages over vision. First, the vestibular organs, located as they are in the inner ear, provide information about movements of the head directly, eliminating the need to convert from eye-centred to head-centred coordinates and avoiding the errors that arise in so doing. Second, the vestibular signal is uncontaminated by the movement of external objects. On the downside, the mechanics of the vestibular organs are such that they provide information about head accelerations rather than velocities (see Angelaki and Cullen, 2008; Lopez and Blanke, 2011 for recent accounts of the vestibular system). The otoliths respond to the linear accelerations that occur during translational body motion and can specify direction of heading. However, the otolith organs also respond to head tilt relative to gravity, such that otolith signals require further processing to represent head translation (Angelaki et al., 2004; Angelaki and Cullen, 2008). Information about speed of heading can be derived from the same linear acceleration signals but this is only possible when acceleration is non-zero; if we move with a constant direction and speed the vestibular system eventually goes quiet. As in the case of vision, the computations required to establish heading from vestibular signals are not trivial, even when good acceleration signals are available. Computing speed is particularly difficult (see for example Laurens et al., 2017) and large systematic errors can occur.

Because the two systems have different limitations, there is scope for each to compensate for the other's deficiencies. Most fundamentally, (i) vision continues to work when accelerations are too small to provide reliable vestibular 
information and (ii) vestibular signals persist when vision is obscured. Most of the time, however, both systems are operative and in these circumstances the two systems provide separate estimates of self-motion that can be combined to provide the best possible overall estimate. Given that both systems are errorprone, the potential for improving perceptual accuracy by cue combination is significant.

The vestibular system also assists vision more directly: at the level of sensory detection. When the head moves, vision is potentially disrupted. When head movements are detected by the vestibular organs, reflexive compensatory movements are initiated that tend to keep the eyes and head stable relative to the external world. An example is the vestibulo-ocular reflex (VOR, e.g., Dieterich \& Brandt, 1995) in which head rotation produces a reflexive counterrotation of the eyes, helping to maintain visual stability. Thus, vestibular cues generate reflexes to reduce unwanted optic flow. Neck proprioception and efference copies of motor signals (von Holst and Mittelstaedt, 1950) also assist.

A full understanding of the sensory processes underlying perception of selfmotion requires descriptions and explanations at different levels: behavioural, neurophysiological and computational. The capabilities and limitations of the visual and vestibular systems in perceiving self-motion have been reviewed previously (Andersen et al., 1999; Angelaki and Cullen, 2008; Angelaki et al., 2009; Bremmer, 2005; Cullen, 2012; Dieterich and Brandt, 2015; Fetsch et al., 2013; Harris, 1994). The literature includes attempts to provide computational descriptions of the relevant sensory processes, which are too complex to be understood fully without considering quantitative aspects. In recent years, extensive new evidence has emerged concerning the vestibular response properties of neurons in various disparate regions of the macaque cerebral cortex, with a focus on visual-vestibular interactions. In parallel, data have emerged from the use of functional magnetic resonance imaging (fMRI) concerning the cortical regions involved in visual-vestibular interactions in humans. The present review focuses on the neuroanatomical substrates of those aspects of visual and vestibular processing that are relevant to perception of selfmotion. A key aim is to identify commonalities and differences between the two species. This is relevant to the difficulties associated with linking primate physiology to human behaviour: not only are the two endeavours conducted at different levels of explanation but linking them ignores any species differences. Although the fundamentals of detection and subcortical processing of vestibular information may be similar in humans and macaques, this is less likely to be true at the cortical level. A helpful aid to linking physiology and behaviour may be to consider the roles of different cortical regions in the two species and their homologies. 


\section{Cortical Candidates for Visual-Vestibular Interaction}

If visual and vestibular signals are to work together to provide a best estimate of self-motion, they must first be brought together in the same brain regions. Although extensive visual and vestibular processing occurs subcortically, the most likely locations for this to occur are cortical. The nature of the interaction between the two senses has received detailed consideration in recent years. A key concept in multisensory cue combination is that cues from different sensory systems may be combined in a way that is dynamic and depends on the reliability of the cues from moment to moment (Ernst and Banks, 2002; Knill and Pouget, 2004). In the case of visual-vestibular cue combination, Fetsch et al. (2009) demonstrated this dynamic quality behaviourally in both macaque and human subjects. Participants made heading judgements that were based on cues from both modalities. The participant was seated in a chair that was moved, by a motion platform, along a straight path with an acceleration-deceleration cycle designed to give good vestibular activity throughout. Participants could not see their environment but instead were presented with an artificial visual scene that simulated linear self-motion having the same acceleration profile. In some trials, the simulated optic flow matched the actual body translation but in others there was a mismatch between the heading directions indicated by the two modalities. It was found that participants made judgements of heading that were intermediate between the two stimulus directions. The reliability of the visual cues was then varied by adding a variable level of noise. In both humans and monkeys, systematically re-weighting towards the vestibular direction occurred as the visual cue became less reliable. Such dynamic cue weighting has also been modeled at the neuronal level. For example, Ohshiro et al. (2011) have provided a model of visual-vestibular integration employing divisive normalization. In each neuron, inputs from two or more senses are passed through a compressive input transformation and then summed with different weights. An expansive output non-linearity is applied to the combined signal and the output is divided by the summed activity of all neurons. The normalization model explains several established features of multisensory integration, including effects of cue reliability on neuronal responses (Morgan et al., 2008). Another interesting feature of cue combination relates to the problem of distinguishing self-motion from object-motion. In a behavioural experiment, Dokka et al. (2015) trained monkeys to report heading direction from optic flow or inertial movement in the presence of a moving visual object. They found that object motion biases visual heading perception (motion is not accurately parsed) but when vestibular heading cues are present, the bias is substantially reduced.

Where in the brain do interactions such as these take place? In the realm of macaque neurophysiology, this question has been tackled primarily by testing 
for vestibular responses in cortical regions already identified as important for the analysis of optic flow. Extensive and detailed data of this kind are now available. In the realm of human fMRI, numerous studies have described visual responses to optic flow but vestibular research is much less advanced. In monkeys, making single-unit recordings during natural vestibular stimulation (passive movement of the animal), although technically demanding, is possible. In humans, fMRI scanning during natural vestibular stimulation is not possible so progress has relied on artificial vestibular stimulation. Two methods are available. The first is galvanic vestibular stimulation, which typically induces a roll sensation, sometimes yaw and pitch rotations, but not linear translation (Fitzpatrick and Day, 2004). The second is caloric vestibular stimulation, which induces a sensation of left-right translational motion or yaw (Dichgans and Brandt, 1978) but cannot be configured to give forward motion, let alone to parametrically vary heading direction. Although several groups have successfully used these methods during fMRI to map the regions of the human brain that are active during vestibular stimulation (Fasold et al., 2002; Frank et al., 2014; Lobel et al., 1998; Smith et al., 2012; Suzuki et al., 2001), measurement of sensitivity to heading direction is not currently possible. Ways forward in the future will perhaps not involve fMRI but will rely on technologies that can be used during natural vestibular stimulation. One possibility is the use of EEG (electroencephalograms), a technique beset with difficulties of interpretation but amenable to improved methods for reliable localization of sources. Otherwise we must await new technical developments in brain imaging, conceivably the refinement of near infrared spectroscopy (NIRS) to a level that permits imaging with a resolution of a few millimetres. Meanwhile, despite the relative paucity of human data, enough is known to begin to ask questions about possible cortical homologies between the macaque and human brains. Here we review the literature by considering each of the main candidate cortical areas in turn. The locations of the cortical regions discussed are shown in Fig. 1.

\section{MSTd}

In macaques, the earliest and most studied cortical region containing neurons that respond selectively to specific types of optic flow is the area known as MSTd (dorsal portion of the medial superior temporal visual area). This region, occupying part of the upper bank of the superior temporal sulcus, is adjacent to the even more highly studied 'motion area' MT (middle temporal area) where motion sensitivity is very strong but thought to be restricted to laminar motion across the receptive field and not to extend to complex flow patterns. Using random-dot patterns that could be configured to simulate natural optic 


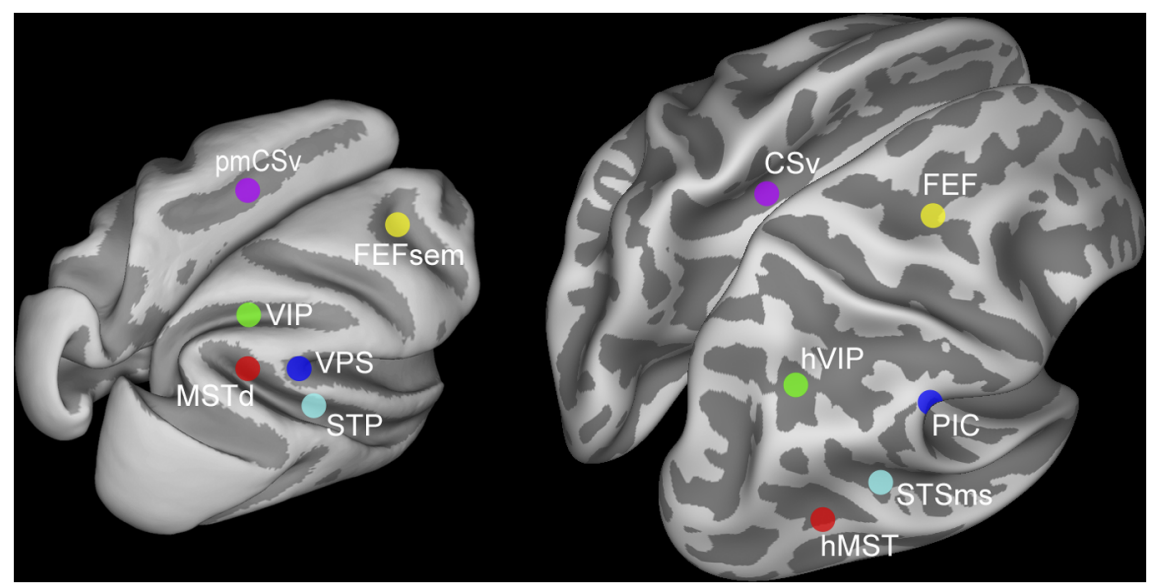

Figure 1. Posterior/lateral view of the cerebral cortex of the macaque (left) and human (right) brains (not to scale). The images are based on structural MRI templates available in CARET (Van Essen et al., 2001) that have been 3D-rendered and partially inflated to expose the sulcal grey matter. In each species, the locations of candidate regions for visual-vestibular interactions are marked. The macaque image is modified from Cottereau et al. (2017). The human image shows approximate locations based on fMRI studies cited in the text. In most of the regions marked, both visual and vestibular responses are known to be present but note that vestibular responses have not so far been documented in macaque STP or pmCSv. Possible homologies between species are indicated by common colours. For clarity, cortical regions are marked in one hemisphere only but all exist bilaterally.

flow patterns, particularly rotation (such as occurs during head roll) and expansion (forward translation), early studies of MSTd (Saito et al., 1986; Tanaka and Saito, 1989; Tanaka et al., 1989) identified neurons with very large receptive fields that are sensitive to the directional structure of the local dot motion array within it. Although tuning was typically broad, different neurons showed clear preferences for different flow structures, suggesting that their function is to analyse optic flow and extract a visual estimate of self-motion. It was posited that MSTd receptive fields are constructed by assembling directionsensitive components manifest as afferents from MT, where receptive fields are smaller. This notion remains valid today (e.g., Mineault et al., 2012), although exactly how such assembly can best be characterized remains a matter of debate. Later studies showed that optic flow preferences form a continuum (Duffy and Wurtz, 1991) that includes spiral motion (Graziano et al., 1994). A property central to the present review is that neurons with a preference for flow patterns that simulate linear translation of the head (e.g., forward, backward, or lateral motion) show a significant degree of tuning for the direction of simulated heading (Duffy and Wurtz, 1995; Page and Duffy, 1999). This makes it likely that MSTd is involved in determining direction of heading during self-motion, a contention supported by the fact that micro-stimulation of 
MSTd can bias behavioural judgements of perceived visual heading in awake monkeys (Britten and Van Wezel, 1998) and that reversible inactivation of MSTd impairs visual heading judgements ( $\mathrm{Gu}$ et al., 2012).

More recently it has been shown that many visually responsive MSTd neurons also respond to vestibular stimulation resulting from whole-body movement in darkness (Duffy, 1998; Gu et al., 2006; Page and Duffy, 2003). Cells are clustered according to vestibular heading preference (Chen et al., 2008), as they are for visual direction (Britten, 1998). The origin of vestibular afferents to MSTd is uncertain but (as is also the case for visual afferents) it is unlikely to be the thalamus or the brainstem. It has been suggested, based on response latency, that vestibular information may reach MSTd by a somewhat circuitous cortical route (Chen et al., 2011a). MSTd may therefore carry highly processed or specialized information. In particular, responses are dominated by velocity rather than acceleration (Fetsch et al., 2010), consistent with processing having occurred to bring vestibular signals into line with visual signals. In addition, vestibular signals in MSTd are specific to translation and are not affected by orientation relative to gravity (Liu and Angelaki, 2009), unlike otolith afferents.

The presence of both visual and vestibular heading signals in the same neurons suggests that MSTd is involved in the process of integrating multisensory cues relevant to perceptual monitoring of self-motion. In the past decade, this suggestion has been confirmed and elaborated; strong evidence now exists for visual-vestibular integration in macaque MSTd. Gu et al. (2006) examined visual and vestibular heading tuning in MSTd neurons in detail. They found that many neurons were tuned to direction of heading in both modalities. When they compared the tuning functions across modalities they found two cell classes that they termed 'congruent' and 'opposite'. Congruent cells had similar heading preferences irrespective of whether heading cues were from inertial motion in darkness or from visual stimulation in the absence of inertial movement. Such cells are clearly well placed to integrate visual and vestibular heading cues. Gu et al. (2007) compared behavioural judgements of inertial heading in darkness with tuning in MSTd and found that heading discrimination performance correlated well with neuron performance, consistent with the notion that vestibular information feeds into heading perception at the level of MSTd. Opposite cells had opposite heading preferences for the two modalities. The function of these neurons is less clear, but recent computational work suggests that they could be used for dissociating self-motion and object motion (Kim et al., 2016). Takahashi et al. (2007) performed similar physiological experiments to those of $\mathrm{Gu}$ et al. (2006) but used visual and vestibular rotations (about various axes in three dimensions) as well as linear translations. They found that many neurons were selectively sensitive to specific vestibular rotations (most often head roll). However, the preferred 
rotation direction was usually opposite to the preferred visual rotation. The paucity of cells with congruent sensitivities would suggest that MSTd does not integrate multi-sensory cues for best perception of rotation but is more likely using vestibular cues to offset confounding effects of head roll when estimating heading. In short, MSTd may be specialized for analyzing heading during locomotion rather than body-motion in general.

Extensive further work has elaborated our understanding of vestibular responses in MSTd and their integration with visual responses. It has proved useful to study the ability of neurons to discriminate fine differences in heading direction in terms of the slope of the tuning function. In congruent cells, visual and vestibular inputs generally sum linearly and this results in a greater slope, and hence greater potential for discrimination ( $\mathrm{Gu}$ et al., 2008). The same authors confirmed behaviourally that discrimination performance is indeed superior when both cues are present. Most cells have vestibular and visual heading tuning profiles that favour directions either left or right of centre, such that tuning functions often have steep slopes around straight-ahead $(\mathrm{Gu}$ et al., 2010). This property could account for the superior behavioural heading discrimination performance found near straight-ahead (Crowell and Banks, 1993). Reliability-based integration of heading cues has been demonstrated in congruent MSTd cells and quantified (Fetsch et al., 2012; Morgan et al., 2008). Further evidence for direct involvement of MSTd in heading perception comes from the finding that behavioural heading judgements can be predicted from the population of congruent MSTd cells (Fetsch et al., 2012), including the dependence of heading percepts on visual cue reliability. Although microstimulation of MSTd can bias visual heading judgements (Britten and Van Wezel, 1998), it has only modest effects on vestibular heading judgements in darkness (Gu et al., 2012). Thus, MSTd is a site for cue integration but with vision taking the lead; presumably vestibular performance in darkness relies more heavily on other cortical areas, such as PIVC or VPS (Chen et al., 2010, 2011b).

Like macaques, humans are able to integrate visual and vestibular cues to self-motion. Heading judgements can be made with reasonable precision based on inertial motion in darkness (Butler et al., 2010; Telford et al., 1995) and also based on visual heading displays without inertial motion (Butler $e t$ al., 2010). When visual simulations and inertial motion are combined, the evidence is limited and mixed but performance may be better than that based on either cue alone, suggesting efficient integration, and there is some evidence that the two cues are weighted dynamically in a way that reflects cue reliability (Butler et al., 2010; Fetsch et al., 2009). It is therefore reasonable to suppose that the two species have similar abilities that operate on similar principles. Unsurprisingly, the neuroanatomical and neurophysiological substrates of these abilities are much less well documented in humans than in macaques. 
In the case of MSTd, a human homologue has been proposed, referred to as hMST (human MST). A complex of cortical visual areas that are highly responsive to motion is readily identifiable with fMRI, located in lateral occipital cortex. This has been referred to as the MT complex, or hMT+, on the assumption that it corresponds loosely to the group of motion-sensitive areas in the posterior/dorsal portion of the macaque superior temporal cortex, including MT and MSTd. A key property of MSTd neurons is that their receptive fields are large, usually include the fovea and are not restricted to the contralateral visual field but commonly extend across the midline to include part of the ipsilateral field (e.g., Komatsu and Wurtz, 1988). This is not the case in MT, where sensitivity is largely confined to the contralateral hemifield. Applying the fMRI equivalent of this criterion to human cortex, it is possible to divide hMT + into two zones, a posterior portion where responses are elicited mainly by contralateral moving stimuli and an anterior portion where strong ipsilateral drive also occurs (Dukelow et al., 2001; Huk et al., 2002). By analogy with macaque MT and MSTd, these areas have become known as hMT and hMST.

Various studies have delivered artificial vestibular stimulation (either galvanic or caloric) during fMRI scanning to map the vestibular regions of the human brain (e.g., Bucher et al., 1998; Lobel et al., 1998; Stephan et al., 2005). Galvanic vestibular stimulation (GVS) involves passing a controlled current between two electrodes located over the mastoid bones behind the two ears, to stimulate the eighth cranial (vestibulocochlear) nerve that carries signals from the inner ear to the brainstem (Fitzpatrick and Day, 2004). Caloric vestibular stimulation, on the other hand, involves inducing a temperature differential between the two ears with warm and/or cold water irrigation, inducing endolymph flow primarily in the horizontal canals and thus causing an imbalance in neural activity (Gernandt, 1949; Barnes, 1995). Studies employing these methods reveal a prominent region of activity in and around the posterior insula, widely regarded as the homologue of macaque parieto-insular vestibular cortex (PIVC) and given the same name. Various other vestibular hotspots have also been identified (see particularly Stephan et al., 2005) but lateral occipital cortex is not prominent among them, although vestibular activity in the vicinity of MT+ is apparent in the data of Bense et al. (2001) and Fasold et al. (2002). In a study specifically targeted at hMT+, Smith et al. (2012) localised hMT and hMST in their participants with the method of Huk et al. (2002) and then delivered galvanic vestibular stimulation during fMRI in darkness. They were careful to control for somatosensory activity arising from the electrode site (where a tingling sensation is typically experienced), a precaution lacking in some earlier studies. When regions of interest were defined corresponding to hMT and hMST and activity within each region was averaged, vestibular activity was clearly seen in hMST but no such activity 
was found in hMT. Thus, the activity previously seen in MT+ arises entirely in hMST. Smith et al. observed that vestibular activity was not spread evenly throughout hMST but was consistently confined to an anterior portion of it. This confirms earlier suspicions, based mainly on knowledge of macaque cortex rather than human fMRI data, that hMST is not a single visual area but contains at least two subregions, only one of which exhibits vestibular responsiveness. Smith et al. (2012) referred to the two areas as hMSTa and hMSTp (anterior and posterior).

The mere presence of vestibular activity in hMSTa does not reveal the nature of the interactions with visual signals that occur there. Indeed, it does not necessarily indicate that the two signals interact at all. A few studies have presented visual and vestibular stimuli together during scanning and documented the brain regions that are responsive (Della-Justina et al., 2015; Deutschländer et al., 2002). However, demonstrating and characterizing interactions between the two signals with imaging techniques is extremely difficult because inertial motion cannot be used and the scope for parametrically manipulating artificial vestibular stimulation is extremely limited. Ideally, one would wish to vary vestibular heading direction and study interactions with simulated visual heading, as has been done in macaques. This is simply not possible with current techniques. Nonetheless, an attempt to quantitatively assess visual-vestibular interactions has been made by Billington and Smith (2015). In this study, galvanic vestibular stimulation was applied while the participant lay supine in an MRI scanner and the resulting head roll sensation was measured psychophysically by nulling it with simulated visual roll. Once the magnitude of visual motion needed for nulling was known, it was used in fMRI experiments in which simulated vestibular and visual roll cues of equal magnitude were presented together, either in the same direction, so that they were experienced as summed, or in opposite directions, so that they cancelled and no roll was experienced. The two combinations elicited about equally strong responses in hMSTa, showing that hMSTa activity reflects the physical stimulus, not the perceptual outcome which was very different in the two cases. Two recent caloric vestibular stimulation studies (Frank et al., 2014; Roberts et al., in press) have also failed to find a difference in hMST response magnitude between congruent and opposite motion, although the use of stimuli that were not matched for motion magnitude across the two senses limits the sensitivity of such analyses. Using multi-voxel pattern analysis, Billington and Smith (2015) were able to predict, or classify, the stimuli based on the responses they elicited in hMSTa, suggesting that congruent and opposite stimulus combinations drive different neuronal populations, at least to some extent. This raises the possibility that two distinct neuron populations exist, tuned for the congruent and opposite directions of roll and potentially used for estimating and discounting head roll signals, respectively. However, roll is a rotation and 
given that macaque MSTd neurons mainly have opposite rather than congruent rotation preferences (Takahashi et al., 2007), an alternative interpretation could be that decoding is possible because one stimulus drives opposite cells and the other drives only neurons that are indifferent to the cross-modal direction relationship: decoding requires only that the two conditions activate non-identical neuron populations. Whether neurons with congruent or opposite preferences for translation (heading) exist in hMSTa is unknown.

Taken together, the results of human fMRI studies and macaque neurophysiology are consistent with a homology between a portion (MSTd) of macaque MST and a portion (hMSTa) of human hMST, both of which receive vestibular as well as visual afferents. However the exact correspondence, if one exists, between subregions of macaque and human MST is extremely uncertain. Most fundamentally, the composition of hMST in terms of subregions is uncertain and so comparison between its subregions and those of macaque MST (and perhaps neighbouring areas such as FST) is, for the moment, precluded. At least two attempts have been made to refine the composition of hMT+. The first (Amano et al., 2009) used retinotopic mapping and identified two maps of the contralateral hemifield that were referred to as TO- 1 and TO-2. The authors did not make an empirical comparison with the above hMT/hMST distinction but they speculated that TO-1 corresponds to hMT. TO-2, located broadly where hMST is expected, bordered TO-1, had a mirror-image field map with receptive field size estimates similar to TO- 1 and a shared foveal representation with TO-1. These properties are suggestive of a homology not with MSTd but instead with the lateral/ventral subdivision of macaque MST, MSTl (also called MSTv). The location of any MSTd homologue is therefore left open. In such a scheme, it would be expected to lie adjacent to TO-2 but the very large receptive fields of macaque MSTd make it unlikely that a human homologue could be located with retinotopic mapping. Using similar retinotopic mapping methodology, Kolster et al. (2010) described four visual areas arranged in a pinwheel arrangement with a shared foveal representation. Using labels from the macaque literature, they refer to them as MT/V5, pMSTv, pV4t and pFST. The first two likely correspond to TO- 1 and TO-2 respectively. The arrangement is similar to that demonstrated by the same group with fMRI in macaques (Kolster et al., 2009). Use of the same methods in both species is invaluable in testing homologies and, in this instance, suggests strong correspondence between the two species but, unfortunately for our purposes, this does not extend to MSTd. The use of structural MRI markers for MT+ has not so far shed any further light: the only study to attempt this shows a high level of variability in the overlap between structural (based on estimates of myelin concentrations) and functional MRI definitions (Large et al., 2016).

To summarize a rather complex situation, there are good reasons to believe that some kind of macaque-human homology exists in relation to MT. The fact 
that hMT does not respond during galvanic stimulation (Smith et al., 2012) and macaque MT neurons do not respond during inertial motion (Chowdhury et al., 2009) is consistent with this proposed homology. There are also some grounds to postulate a homology in relation to MSTl (MSTv). It is plausible that one also exists in relation to MSTd. TO-2 is immediately anterior to TO1 (hMT) suggesting that TO-2 might correspond to hMSTp of Smith et al. (2012). hMSTa, where vestibular activity is found, is immediately anterior to hMSTp and such a location is plausible for an MSTd homolog. Such an arrangement would apparently leave human MSTd without the border with MT that is described in the macaque literature (Desimone and Ungerleider, 1986) but that border is at the representation of the far periphery of the visual field and might become evident with a sufficiently large visual stimulus. Currently an MSTd-hMSTa homology is a good guess but may prove incorrect.

\section{VIP/hVIP}

The macaque ventral intraparietal area, VIP (Colby et al., 1993), is a polysensory area in the fundus of the intraparietal sulcus that encodes visual, vestibular, auditory and somatosensory stimuli. It is particularly sensitive to visual stimuli located near the observer in depth (Bremmer et al., 2013; Colby et al., 1993; Yang et al., 2011) and to air puffs on the face (Avillac et al., 2005), suggesting that it may be specialised for encoding nearby objects such as reach targets and/or obstacles during locomotion. There is evidence not only for visual-vestibular integration (discussed below), but also for visualauditory (Schlack et al., 2005) and visual-somatosensory (Avillac et al., 2007) integration.

Most VIP neurons respond well to at least one type of optic flow (Bremmer et al., 2002a; Schaafsma and Duysens, 1996), some preferring expansion or contraction, some rotation and some translation. Of those that prefer expansion, most are sensitive to heading direction, indexed by the location of the focus of expansion. As in MSTd, they are quite broadly tuned, but selective enough to support perceptual heading judgements (Zhang and Britten, 2010). Indeed, responses to optic flow in VIP appear similar to those in MSTd in most respects (Chen et al., 2011a). VIP is connected with MST (Boussaoud et al., 1990), which may be a key source of visual input and account for the similarity of its visual response properties, although response latencies are similar in the two areas consistent with parallel inputs from some other source (Chen $e t$ al., 2011c).

There is strong evidence for visual-vestibular integration in macaque VIP. Vestibular drive was first demonstrated in VIP by Bremmer et al. (2002b) who observed that visual and vestibular responses of heading-selective neurons were typically tuned for the same axis of translation. As in MSTd, some 
cells have congruent heading preferences and some opposite (Chen et al., 2011c; Schlack et al., 2002). In congruent cells, heading sensitivity is similar to congruent MSTd cells and it is greater during bimodal stimulation than with either stimulus alone, suggesting cue combination (Chen et al., 2013a). As in MSTd, optic flow tuning is in eye-centred coordinates (Chen et al., 2013b). In contrast, vestibular translation tuning is organized within a head-centred reference frame (Chen et al., 2013b). About half of VIP neurons are able to encode heading and eye rotation (including that arising from smooth pursuit eye movements) in a separable manner (Sunkara et al., 2016).

A polysensory cortical region has also been identified in the fundus of the human intraparietal sulcus. The initial description (Bremmer et al., 2001) showed clearly with fMRI that visual, auditory and somatosensory responses are present; vestibular sensitivity was not tested. Strong grounds for considering this region homologous with macaque VIP were claimed and accordingly, we refer to this region as hVIP (human VIP). Selectivity for motion that arises from self-motion is greater in hVIP than in hMST, as evidenced by comparing the response to a large optic flow pattern with that to an array of nine flow patterns, each of which could be interpreted as self-motion but only when considered alone (Wall and Smith, 2008). In hVIP, the response is reduced by about half when the stimulus is made incompatible with self-motion in this way. In hMST, the response is also reduced, but much less. In a recent macaque fMRI study using the same stimuli (Cottereau et al., 2017), a similar pattern of results was found: about 50\% reduction in VIP activity for a nine-patch array but a smaller reduction in MSTd, consistent with the VIP homology claimed by Bremmer et al. (2001) and also with a homology at the level of MSTd.

The vestibular status of hVIP is less clear than that of hMST. In a study that measured vestibular activity in regions of interest defined with visual localisers (Smith et al., 2012), there was a hint of vestibular activity in visually defined hVIP, but it was small and not significantly different from zero. The region examined was at a very similar location to hVIP of Bremmer et al. (2001). Based on this study, vestibular activity is seemingly weaker in hVIP than in macaque VIP, or even absent. A logical possibility is that hVIP has vestibular sensitivity but that this does not include sensitivity to the head roll that dominates the sensation induced by galvanic stimulation. However, a similar weakness of hVIP activity is present in the data of Frank et al. (2016a) who used caloric stimulation, which induces primarily a sensation of yaw rotation or horizontal translation. They were able to find vestibular activity in hVIP only when a very liberal threshold was used (personal communication). Although there is thus no clear and direct demonstration of vestibular activity in hVIP, less direct evidence comes from the results of Billington and Smith (2015), described above in connection with hMST. These authors were able to decode congruent and opposite roll motions in hVIP as well as in hMSTa (and 
in PIC, see below). Although they did not test vestibular stimulation in darkness, this result shows indirectly that vestibular sensitivity must exist in hVIP. Moreover, vestibular responses have been reported (Stephan et al., 2005) at a location described as inferior parietal lobule but with coordinates that were close to the expected location of hVIP. If this is indeed hVIP, which is uncertain, the elusive nature of hVIP vestibular activity in the only study to use a region-of-interest approach (Smith et al., 2012) is unexplained and possibly due simply to limitations of measurement sensitivity. At best, roll sensitivity is demonstrable; unfortunately it is not possible to configure galvanic stimulation to induce forward motion of the kind needed to test for heading responses. If vestibular heading signals do exist then it becomes likely that they interact with visual signals in the way that occurs in macaque VIP, but this is untested.

Although the convergence of visual, auditory, somatosensory and possibly vestibular responses in hVIP does suggest a homology with macaque VIP, care is needed because the organization of the intraparietal sulcus is different in the two species. In humans, there are several more visual areas in and around the sulcus than are thought to exist in macaques (Orban et al., 2006; Swisher et al., 2007). It remains possible nonetheless that one of them corresponds to VIP and that a straightforward functional homology exists. An additional reason for caution is that the preference for near visual stimuli found in macaque VIP is apparently absent in hVIP (Quinlan and Culham, 2007). The failure to find it could reflect methodological limitations but the same study successfully found such a preference in the parieto-occipital sulcus.

\section{VPS/PIC}

The core vestibular region in the macaque cerebral cortex is thought to be PIVC. PIVC receives direct vestibular input from the vestibular nuclei through the thalamus and is sometimes regarded as primary vestibular cortex. PIVC is not a likely candidate for visual-vestibular integration because most PIVC neurons do not exhibit excitatory visual responses (Chen et al., 2010). However, immediately posterior to PIVC lies a separate cortical region that, like PIVC, responds to both rotations and translations in darkness. This region is distinguishable from PIVC in that it also responds well to optic flow (Chen et al., 2011b). These authors refer to this area as VPS (visual posterior Sylvian) but in earlier literature (e.g., Guldin et al., 1992) it was called the parietotemporal association area T3. It is strongly connected with PIVC, from which it presumably receives vestibular signals, and also receives a projection from MSTd, a likely source of optic flow signals (Guldin and Grüsser, 1998). Many VPS neurons respond selectively to heading direction in both modalities. As in MSTd and VIP, their preferred translation axes usually align between the two modalities and both congruent and opposite heading preferences are found. 
However, opposite preferences strongly dominate. Consequently, VPS may prove to be a key site for some forms of visual-vestibular interactions but perhaps not for optimizing perception of self-motion trajectory.

PIVC has the same status in humans, with direct subcortical vestibular inputs recently characterised in detail (Kirsch et al., 2016). Moreover, a likely human homolog of VPS has been identified in humans (Frank et al., 2014; Frank et al., 2016a). Several imaging studies had previously identified visual responses at a location very close to PIVC, so close that sometimes such responses were misinterpreted as visual responses in PIVC itself. The region was named PIC (posterior insular cortex) by Sunaert et al. (1999) based on responsiveness to visual motion. Confirmation that PIVC and PIC are distinct comes from a recent diffusion tensor imaging study (Wirth et al., subm.) showing different connectivity patterns: PIC exhibits predominant connectivity to superior temporal sulcus (STS), to the supramarginal gyrus, and to the intraparietal cortex, whereas PIVC exhibits predominant connectivity to areas in the anterior insula and precuneus. It was recently shown that engaging in a visual task enhances visual activity in PIC compared to passive viewing of the same stimuli (Frank et al., 2016b). It has also recently become clear that PIC responds to vestibular stimuli. Frank et al. (2014) identified PIC with a visual localiser and then sought vestibular responses within it by delivering caloric vestibular stimulation during fMRI scanning. They found robust vestibular responses in near-darkness, as well as responses to optic flow in the absence of vestibular stimulation. Both stimuli were then presented together in two arrangements: caloric stimulation usually causes left-right movement sensations and they therefore added visual left-right flow. Combining the two stimuli in either congruent or opposite directions yielded responses of similar magnitude. Although opposite motion gave slightly larger responses, as would be predicted if there was a preponderance of opposite cells as in VPS, the difference was not significant. However, Billington and Smith (2015) were able to decode congruent and opposite visual-vestibular roll combinations in PIC, suggesting orderly visual-vestibular interactions. Thus far, the evidence for a homology between VPS and PIC is encouraging.

\section{FEFsem}

One further cortical area in macaques has been shown to contain multisensory visual-vestibular neurons like those in MSTd and VIP that show tuned responses and matched preferences: FEFsem, the division of the frontal eye fields associated with the control of smooth pursuit eye movements. Many FEFsem cells show significant heading sensitivity in both modalities and again, both congruent and opposite heading preferences are present ( $\mathrm{Gu}$ et al., 2016). Given that FEFsem is regarded as having oculomotor rather than 
perceptual functions, it is likely that visual-vestibular interactions exist in the region for motor rather than perceptual purposes. More specifically, $\mathrm{Gu}$ et al. (2016) suggest that FEFsem may play a prominent role in coordinating volitional eye movements during self-motion.

The existence of human frontal eye fields is well established and has been confirmed with fMRI, although human FEF has been studied (e.g., Corbetta $e t$ al., 1998; Kimmig et al., 2001; Luna et al., 1998) and compared to macaque FEF (Koyama et al., 2004) mainly in the context of saccades rather than smooth pursuit. It has however been shown to be active during smooth pursuit (Tanabe et al., 2002). Direct comparison between activations associated with saccades and pursuit have been made (Petit et al., 1997), suggesting that pursuit is associated with more ventral locations of activation in the FEF. However, Berman et al. (1999) point to a high overlap in the patterns of activations evoked by saccades and pursuit. Differences in the results of these two studies could be related to the frequency of small 'catch-up' saccades evoked during pursuit, which serve to re-centre the fovea on the pursuit target (Haller et al., 2008). Kimmig et al. (2008) compared fMRI activations during passive viewing of a moving single dot with activation when subjects pursued a single dot. Appropriate contrasts compared the oculomotor, sensory and combined effects on fMRI activation in the FEF, the supplementary eye fields (SEF), posterior parietal cortex (PPC), MSTd, and primary visual cortex (V1). Combined visual-motor stimulation led to the most pronounced activations in all of these areas. In summary, there is some support for a subdivision of the human frontal eye fields in the control of saccadic and smooth pursuit eye movements. However, this evidence is not particularly strong. Vestibular activity in human FEF has been reported in fMRI studies involving artificial vestibular stimulation (Bense et al., 2001, Stephan et al., 2005), although this activity could be related to the reflexive eye movements that are induced by vestibular stimulation, rather than to the analysis of self-motion. Responses to visual motion have been observed in FEF (e.g., Frank et al., 2016b) but specificity for self-motion has not been documented. It is thus uncertain whether human FEF performs highly developed visual-vestibular interactions akin to those that have been observed in macaque FEFsem.

\section{7. $\mathrm{CSv}$}

In the human cortex, a new focus of self-motion processing has recently emerged (Wall and Smith, 2008): the cingulate sulcus visual area (CSv). This region responds well to optic flow stimuli but not to random motion (Antal et al., 2008), with one study claiming that it is actually suppressed by random motion and also by static visual stimuli (Pitzalis et al., 2013a). Indeed, even with an optimal optic flow stimulus, the excitatory response is quite small as 
measured with fMRI. The strongest responses are obtained with dynamically changing optic flow, such as a stimulus that cycles through spiral space from expansion to rotation and back (Wall and Smith, 2008) or a heading stimulus in which the direction of heading is continuously changing (Furlan et al., 2014). Responses to unchanging flow such as continuous expansion or continuous horizontal translation are dramatically smaller in CSv. Despite the weakness of the response in comparison to, say, hMST or hVIP, CSv has a striking feature. When the paradigm of replacing optic flow with an array of optic flow patches is applied (see hVIP above), the response in CSv is almost completely abolished (Wall and Smith, 2008; Cardin and Smith, 2010). An array of optic flow patches is apparently met with the same indifference as random motion, the feature the two have in common being that neither indicates that self-motion is occurring. Other areas (hVIP, hV6 and to a limited extent hMST) show signs of this behaviour but CSv shows it much more strongly than any other area examined and, if the interpretation is correct, $\mathrm{CSv}$ is therefore much more sensitive to whether a given motion stimulus arises from self-motion.

In additional to these visual responses, compelling vestibular activity has been demonstrated in CSv with artificial vestibular stimuli applied in conjunction with fMRI (Smith et al., 2012). CSv is therefore potentially a site of visual-vestibular interaction. Interestingly, however, the evidence for interaction, such as it is, is negative. Attempts to decode congruent and opposite visual-vestibular combinations (Billington and Smith, 2015) show a complete failure to do so in CSv, despite good success in hMST, hVIP and PIC in the same study. Thus CSv is undoubtedly bi-sensory in humans but evidence for integration of signals is so far lacking. A recent study of the connectivity of CSv (Smith et al., in press) has revealed strong connectivity with nearby cingulate motor areas and also with more distal motor areas including the supplementary motor area. Connectivity with sensory cortex is relatively weak, although PIC and hVIP both emerge as possible sources of sensory afferents. This, together with the near-silence of CSv in the presence of visual motion that does not reflect self-motion, suggests that the role of CSv may be to feed information about self-motion into the motor system. The apparent lack of integration could indicate that disparate sensory signals are merely marshalled for that purpose, rather than integrated, in CSv. There is no particular reason to think that activity in CSv influences conscious perception, although it is possible. There are parallels here with hV6 (see below), which also probably has the primary role of guiding action, but quite different parts of the motor system are involved in the two cases.

Macaque neurophysiology has not yet identified a counterpart of CSv. Neurophysiological exploration of the cingulate cortex in general has been limited, at least partly because of the technical difficulty of recording so deep in the brain without causing damage. A few studies have reported visual activity in 
posterior cingulate regions that could possibly correspond to CSv (Dean et al., 2004; Olson et al., 1993). One study (Guldin et al., 1992; see also Guldin and Grüsser, 1998) has identified a vestibular cingulate region in squirrel monkey. It is possible that this region also has visual sensitivity and constitutes a squirrel monkey counterpart of CSv but this is far from certain. Very recently, the use of fMRI in macaques has tentatively identified a macaque homologue of CSv (Cottereau et al., 2017). In this study, the 1-patch and 9-patch stimuli of Wall and Smith (2008) were presented to fixating monkeys during fMRI and the responses contrasted. A quite similar set of cortical regions emerged to that seen in humans, including differential activity in a small region of the cingulate sulcus that the authors refer to as pmCSv (putative macaque CSv). As in humans, the response in this region to a large changing flow field is reduced almost to zero when the stimulus is replaced by an array of changing flow stimuli. Whether pmCSv also possesses vestibular sensitivity is unknown; to the best of our knowledge no macaque fMRI studies have used vestibular stimulation. Uniquely among the cortical areas reviewed here, human work is more advanced than macaque in the case of CSv.

\section{STP/STSms}

In macaques, a superior temporal polysensory area (STP) has been identified where neurons may respond to visual, auditory and somatosensory stimuli (Bruce et al., 1981; Hikosaka et al., 1988). It is connected with MST (Boussaoud et al., 1990) and many neurons respond well to optic flow, often with a clear preference, most commonly for expansion (Anderson and Siegel, 1999). However visual heading sensitivity in STP neurons has not received the detailed treatment accorded to MSTd, VIP and VPS. In humans, a polysensory region has been identified in the superior temporal sulcus (STS) that may well be homologous with macaque STP (Beauchamp et al., 2004, 2008). It responds to visual, auditory and tactile stimuli and is referred to by Beauchamp's group as STSms (STS multisensory).

Macaque STP, although certainly multisensory, is not known as a vestibular area. However, human STSms responds strongly to artificial vestibular stimulation (Smith et al., 2012) and appears to be well connected to PIVC and PIC (Wirth et al., subm.). Responses to optic flow are sometimes seen but are more elusive, suggesting that vestibular inputs may be stronger than visual. In humans, STSms is therefore a potential site of visual-vestibular interaction. It is not known whether macaque STP has vestibular inputs that have been overlooked or, alternatively, whether their absence reflects a species difference. 


\section{Mono-Sensory Areas Involved in Encoding Self-Motion}

Two further cortical regions are known to be responsive to sensory cues to self-motion but apparently do not receive both visual and vestibular afferents. The first is PIVC, already discussed. PIVC is well documented in primates and is prominent in almost every human imaging study that involves artificial vestibular stimulation. It is a core vestibular area and it is likely that there is a broad homology between macaque and human. However, it does not exhibit excitatory responses to visual stimuli in either species and so it cannot be considered a site of visual-vestibular integration. Indeed, imaging studies suggest that visual stimulation causes neural suppression in human PIVC (Brandt et al., 1998; Frank et al., 2016a) and recently it has been shown that when participants perform a visual-motion tracking task, suppression in PIVC scales with the demands of the task (Frank et al., 2016b). These findings are consistent with generalized and attention-dependent suppressive competition between the two senses.

The second and less obvious region in this category is V6, located in the parieto-occipital sulcus (POS). Earlier neurophysiological work had identified visual responses in this vicinity but macaque V6 was fully characterised relatively late in the history of visual neuroscience. It forms a discrete retinotopically organised visual region with high sensitivity to moving stimuli and strong direction selectivity on the anterior bank of the sulcus (Galletti et al., 1991, 1999). Human V6, located on the posterior bank of the POS but thought to be functionally homologous because of its sensory properties, is also retinotopically organised. Like its macaque counterpart (Colby et al., 1988), it emphasises the peripheral visual field and it requires wide-field stimulation for optimal activation (Pitzalis et al., 2006). Like many motion-sensitive cortical regions it is more responsive to coherent motion than to motion noise (Pitzalis et al., 2010) and indeed is part of a much smaller set of regions (the others being VIP, CSv and PIC) that are more responsive to wide-field optic flow than to an array of optic flow patches (Cardin and Smith, 2010). Human V6 responds well to large expanding, rotating and spiralling optic flow patterns but also responds well to translation (Pitzalis et al., 2013a). The sensitivity of macaque V6 neurons to optic flow stimuli has been examined only recently. Most cells respond well to optic flow and are selective for heading direction, much like those found in MSTd and VIP (Fan et al., 2015). Overall, there is a very good case for a homology between macaque and human V6. A key shared property contributing to this view is that V6, uniquely among cortical visual areas specialised for encoding optic flow, does not respond to vestibular stimulation in either species. Macaque V6 neurons do not respond during inertial motion in darkness even though they mostly respond well to optic flow (Fan et al., 2015) while galvanic vestibular stimulation does not elicit activity in human 
V6 (Smith et al., 2012). Unless something important has been missed, V6 is not a site of visual-vestibular integration in either species. It is apparently a purely visual area that is maximally sensitive to stimuli in near space (Quinlan and Culham, 2007) and it is sensitive to the stereoscopic depth structure (only useful for nearby stimuli) of optic flow (Cardin and Smith, 2011). It may therefore process self-motion in order to aid perception of objects (Pitzalis et al., $2013 \mathrm{~b}$ ), rather than to facilitate perception of the self-motion itself. Its location, close to parietal regions concerned with motor functions such as reaching and pointing, suggests that it may feed visual information about nearby objects into the motor system, either for reaching or for object-avoidance during locomotion, probably via adjacent area V6A (Galletti et al., 1996; Pitzalis et al., 2013c).

\section{Why so Many Cortical Regions?}

It might be expected that a single cortical area specialised for visual-vestibular integration would be sufficient to account for self-motion perception, but it is apparent from the above discussion that there are at least four such areas in both the macaque and human brains. A possible interpretation is that these areas are specialised for different purposes, in which case we would expect this to be reflected in different response properties. However, at least in macaque, a number of response properties that have been examined appear to be rather similar across areas. Heading tuning is similar in MSTd, VIP, PIVC and VPS and this is true for visual, vestibular and combined stimulation (Chen et al., 2011a, 2011b, 2013a). A few identifiable differences exist, however. The simplest lies in the relative dominance of the two senses in each area. In MSTd, visual responses are stronger than vestibular. In VPS, vestibular responses are stronger and in VIP they are relatively balanced. Another difference lies in the frame of reference in which vestibular signals are encoded. In MSTd, vestibular heading tuning, measured in the coordinates of the external world, is influenced by head-on-body position, such that most MSTd neurons signal vestibular heading in a head-centred reference frame (Fetsch et al., 2007). In VIP, vestibular heading tuning is affected by neither eye nor head position, such that heading is represented in body-centred (or perhaps world-centred) coordinates (Chen et al. 2013c; Zhang et al., 2004). Arguably, therefore, some aspects of processing are more evolved in VIP than in MSTd. VIP is also more multisensory than MSTd, showing somatosensory and auditory responses that appear to be absent in MSTd. Another difference among areas involves the dynamics of vestibular heading signals. Whereas PIVC neurons show response dynamics that primarily reflect acceleration of the head, MSTd neurons predominantly represent velocity, with VIP being intermediate (Chen et al., 2011a). However, the differences in vestibular dynamics between 
these cortical areas are modest compared to the substantial transformation that occurs from otolith afferents to the vestibular nucleus (Laurens et al., 2017).

An interesting recent discovery is that MSTd and VIP differ in terms of the effect of inactivation on heading perception. When MSTd is reversibly inactivated with muscimol, visual heading judgements are substantially affected but vestibular heading sensitivity is only modestly disrupted (Gu et al., 2012), suggesting that MSTd may play a greater role in visual heading perception (see also Britten and Van Wezel, 1998). Surprisingly, perhaps, inactivation of VIP results in neither visual nor vestibular heading perception deficits (Chen et al., 2016), despite the fact that VIP neurons show much stronger choice-related activity than MSTd neurons (Chen et al., 2013a). This suggests that VIP may not be involved in perception of heading per se, and may be consistent with the view that VIP is more concerned with obstacles and objects in near-space, for motor rather than perceptual purposes. The strongest effects of inactivation on vestibular heading perception have been found in area PIVC (Chen et al., 2016).

While the existing literature from macaque studies suggests some functional differences between areas, a complete understanding of the respective roles of these cortical areas in self-motion perception remains elusive. This may result, at least in part, from our incomplete understanding of how neural representations of self-motion account for object motion, as well as eye and head movements of the observer. These present substantial computational challenges, and it seems likely that self-motion signals need to be combined with other sensory and motor signals, in a variety of ways, to generate representations of self-motion that are robust in natural environments.

In humans, less information is available about differences between areas. Sensitivity to optic flow structure has been demonstrated in hMST with fMRI adaptation (Wall et al., 2008) but no fMRI studies have so far addressed heading tuning in this or any other area. hMST is more responsive to visual than vestibular stimuli, as in macaques, but this is also true in hVIP (Smith et al., 2012) whereas in macaques the balance is more even. Limited data on the pathways connecting the various visual/vestibular areas are available from recent studies on the connectivity of CSv (Smith et al., in press) and PIC/PIVC (Wirth et al., subm.). The former study suggests that CSv is strongly connected with PIC and hVIP and that these two areas are therefore likely sources of visual and vestibular afferents. Area hV6 is an additional likely source of visual information. The latter study suggests that PIC is more connected to visual and posterior parietal areas, whereas PIVC shows connectivity that is more pronounced in anterior insula and premotor cortex. Beyond this, little is known. 


\section{References}

Amano, K., Wandell, B. A. and Dumoulin, S. O. (2009). Visual field maps, population receptive field sizes, and visual field coverage in the human MT+ complex, J. Neurophysiol. 102, 2704-2718.

Andersen, R., Shenoy, K., Snyder, L., Bradley, D. and Crowell, J. (1999). The contributions of vestibular signals to the representations of space in the posterior parietal cortex, Ann. N. Y. Acad. Sci. 871, 282-292.

Anderson, K. C. and Siegel, R. M. (1999). Optic flow selectivity in the anterior superior temporal polysensory area, STPa, of the behaving monkey, J. Neurosci. 19, 2681-2692.

Angelaki, D. E. and Cullen, K. E. (2008). Vestibular system: the many facets of a multimodal sense, Annu. Revi. Neurosci. 31, 125-150.

Angelaki, D. E., Shaikh, A., Green, A. and Dickman, J. D. (2004). Neurons compute internal models of the physical laws of motion, Nature 430, 560-564.

Angelaki, D. E., Klier, E. M. and Snyder, L. H. (2009). A vestibular sensation: probabilistic approaches to spatial perception, Neuron 64, 448-462.

Antal, A., Baudewig, J., Paulus, W. and Dechent, P. (2008). The posterior cingulate cortex and planum temporale/parietal operculum are activated by coherent visual motion, Vis. Neurosci. 25, 17-26.

Avillac, M., Denève, S., Olivier, E., Pouget, A. and Duhamel, J.-R. (2005). Reference frames for representing visual and tactile locations in parietal cortex, Nat. Neurosci. 8, 941-949.

Avillac, M., Hamed, S. B. and Duhamel, J.-R. (2007). Multisensory integration in the ventral intraparietal area of the macaque monkey, J. Neurosci. 27, 1922-1932.

Barnes, G. (1995). Adaptation in the oculomotor response to caloric irrigation and the merits of bithermal stimulation, Br. J. Audiol. 29, 95-106.

Beauchamp, M. S., Lee, K. E., Argall, B. D. and Martin, A. (2004). Integration of auditory and visual information about objects in superior temporal sulcus, Neuron 41, 809-823.

Beauchamp, M. S., Yasar, N. E., Frye, R. E. and Ro, T. (2008). Touch, sound and vision in human superior temporal sulcus, NeuroImage 41, 1011-1020.

Bense, S., Stephan, T., Yousry, T. A., Brandt, T. and Dieterich, M. (2001). Multisensory cortical signal increases and decreases during vestibular galvanic stimulation (fMRI), J. Neurophysiol. 85, 886-899.

Berman, R. A., Colby, C. L., Genovese, C. R., Voyvodic, J. T., Luna, B., Thulborn, K. R. and Sweeney, J. A. (1999). Cortical networks subserving pursuit and saccadic eye movements in humans: an FMRI study, Hum. Brain Mapp. 8, 209-225.

Billington, J. and Smith, A. T. (2015). Neural mechanisms for discounting head-roll-induced retinal motion, J. Neurosci. 35, 4851-4856.

Boussaoud, D., Ungerleider, L. G. and Desimone, R. (1990). Pathways for motion analysis: cortical connections of the medial superior temporal and fundus of the superior teemporal visual areas of the macaque, J. Comp. Neurol. 296, 462-495.

Brandt, T., Bartenstein, P., Janek, A. and Dieterich, M. (1998). Reciprocal inhibitory visualvestibular interaction - visual motion stimulation deactivates the parieto-insular vestibular cortex, Brain 121, 1749-1758.

Bremmer, F. (2005). Navigation in space - the role of the macaque ventral intraparietal area, J. Physiol. 566, 29-35. 
Bremmer, F., Schlack, A., Shah, N. J., Zafiris, O., Kubischik, M., Hoffmann, K., Zilles, K. and Fink, G. R. (2001). Polymodal motion processing in posterior parietal and premotor cortex: a human fMRI study strongly implies equivalencies between humans and monkeys, Neuron 29, 287-296.

Bremmer, F., Duhamel, J. R., Hamed, S. B. and Graf, W. (2002a). Heading encoding in the macaque ventral intraparietal area (VIP), Eur. J. Neurosci. 16, 1554-1568.

Bremmer, F., Klam, F., Duhamel, J. R., Hamed, S. B. and Graf, W. (2002b). Visual-vestibular interactive responses in the macaque ventral intraparietal area (VIP), Eur. J. Neurosci. 16, $1569-1586$.

Bremmer, F., Schlack, A., Kaminiarz, A. and Hoffmann, K. (2013). Encoding of movement in near extrapersonal space in primate area VIP, Front. Behav. Neurosci. 7, 8. DOI:10.3389/ fnbeh.2013.00008.

Britten, K. H. (1998). Clustering of response selectivity in the medial superior temporal area of extrastriate cortex in the macaque monkey, Vis. Neurosci. 15, 553-558.

Britten, K. H. and Van Wezel, R. J. A. (1998). Electrical microstimulation of cortical area MST biases heading perception in monkeys, Nat. Neurosci. 1, 59-63.

Bruce, C., Desimone, R. and Gross, C. G. (1981). Visual properties of neurons in a polysensory area in seperior temporal sulcus of the macaque, J. Neurophysiol. 46, 369-384.

Bucher, S., Dieterich, M., Weismann, M., Weiss, A., Zink, R., Yousry, T. and Brandt, T. (1998). Cerebral functional magnetic resonance imaging of vestibular, auditory and nociceptive areas during gavanic stimulation, Ann. Neurol. 44, 120-125.

Butler, J. S., Smith, S. T., Campos, J. L. and Bülthoff, H. H. (2010). Bayesian integration of visual and vestibular signals for heading, J. Vis. 10, 23. DOI:10.1167/10.11.23.

Cardin, V. and Smith, A. T. (2010). Sensitivity of human visual and vestibular cortical regions to egomotion-compatible visual stimulation, Cereb. Cortex 20, 1964-1973.

Cardin, V. and Smith, A. T. (2011). Sensitivity of human visual cortical area V6 to stereoscopic depth gradients associated with self-motion, J. Neurophysiol. 106, 1240-1249.

Chen, A., Gu, Y., Takahashi, K., Angelaki, D. E. and DeAngelis, G. C. (2008). Clustering of self-motion selectivity and visual response properties in macaque area MSTd, J. Neurophysiol. 100, 2669-2683.

Chen, A., DeAngelis, G. C. and Angelaki, D. E. (2010). Macaque parieto-insular vestibular cortex: responses to self-motion and optic flow, J. Neurosci. 30, 3022-3042.

Chen, A., DeAngelis, G. C. and Angelaki, D. E. (2011a). A comparison of vestibular spatiotemporal tuning in macaque parietoinsular vestibular cortex, ventral intraparietal area, and medial superior temporal area, J. Neurosci. 31, 3082-3094.

Chen, A., DeAngelis, G. C. and Angelaki, D. E. (2011b). Convergence of vestibular and visual self-motion signals in an area of the posterior Sylvian fissure, J. Neurosci. 31, 11617-11627.

Chen, A., DeAngelis, G. C. and Angelaki, D. E. (2011c). Representation of vestibular and visual cues to self-motion in ventral intraparietal cortex, J. Neurosci. 31, 12036-12052.

Chen, A., DeAngelis, G. C. and Angelaki, D. E. (2013a). Functional specializations of the ventral intraparietal area for multisensory heading discrimination, J. Neurosci. 33, 35673581.

Chen, X., DeAngelis, G. C. and Angelaki, D. E. (2013b). Eye-centered representation of optic flow tuning in the ventral intraparietal area, J. Neurosci. 33, 18574-18582.

Chen, X., DeAngelis, G. C. and Angelaki, D. E. (2013c). Diverse spatial reference frames of vestibular signals in parietal cortex, Neuron 80, 1310-1321. 
Chen, A., DeAngelis, G. C. and Angelaki, D. E. (2016). Evidence for a causal contribution of macaque vestibular, but not intraparietal, cortex to heading perception, J. Neurosci. 36, 3789-3798.

Chowdhury, S. A., Takahashi, K., DeAngelis, G. C. and Angelaki, D. E. (2009). Does the middle temporal area carry vestibular signals related to self-motion? J. Neurosci. 29, 12020-12030.

Colby, C., Gattas, R., Olson, R. and Gross, C. (1988). Topographical organization of cortical afferents to extrastriate visual area PO in the macaque: a dual tracer study, J. Comp. Neurol. 269, 392-413.

Colby, C. L., Duhamel, J. R. and Goldberg, M. E. (1993). Ventral intraparietal area of the macaque: anatomic location and visual response properties, J. Neurophysiol. 69, 902-914.

Corbetta, M., Akbudak, E., Conturo, T. E., Snyder, A. Z., Ollinger, J. M., Drury, H. A., Linenweber, M. R., Petersen, S. E., Raichle, M. E., Van Essen, D. C. and Shulman, G. L. (1998). A common network of functional areas for attention and eye movements, Neuron 21, 761773.

Cottereau, B. R., Smith, A. T., Rima, S., Fize, D., Héjja-Brichard, Y., Renaud, L., Lejards, C., Vayssière, N., Trotter, Y. and Durand, J. B. (2017). Processing of egomotion-consistent optic flow in the rhesus macaque cortex, Cereb. Cortex 27, 330-343.

Crowell, J. A. and Banks, M. S. (1993). Perceiving heading with different retinal regions and types of optic flow, Percept. Psychophys. 53, 325-337.

Cullen, K. E. (2012). The vestibular system: multimodal integration and encoding of selfmotion for motor control, Trends Neurosci. 35, 185-196.

Dean, H. L., Crowley, J. C. and Platt, M. L. (2004). Visual and saccade-related activity in macaque posterior cingulate cortex, J. Neurophysiol. 92, 3056-3068.

Della-Justina, H. M., Gamba, H. R., Lukasova, K., Nucci-da-Silva, M. P., Winkler, A. M. Jr and Amaro, E. (2015). Interaction of brain areas of visual and vestibular simultaneous activity with fMRI, Exp. Brain Res. 233, 237-252.

Desimone, R. and Ungerleider, L. G. (1986). Multiple visual areas in the caudal superior temporal sulcus of the macaque, J. Comp. Neurol. 248, 164-189.

Deutschländer, A., Bense, S., Stephan, T., Schwaiger, M., Brandt, T. and Dieterich, M. (2002). Sensory system interactions during simultaneous vestibular and visual stimulation in PET, Hum. Brain Mapp. 16, 92-103.

Dichgans, J. and Brandt, T. (1978). Visual-vestibular interaction: effects on self-motion perception and postural control, in: Handbook of Sensory Physiology, Vol. 8, R. Held, H. Leibowitz and H.-L. Teuber (Eds), pp. 755-804. Springer, Berlin, Germany.

Dieterich, M. and Brandt, T. (1995). Vestibulo-ocular reflex, Curr. Opin. Neurol. 8, 83-88.

Dieterich, M. and Brandt, T. (2015). The bilateral central vestibular system: its pathways, functions, and disorders, Ann. N. Y. Acad. Sci. 1343, 10-26.

Dokka, K., DeAngelis, G. C. and Angelaki, D. E. (2015). Multisensory integration of visual and vestibular signals improves heading discrimination in the presence of a moving object, J. Neurosci. 35, 13599-13607.

Duffy, C. J. (1998). MST neurons respond to optic flow and translational movement, J. Neurophysiol. 80, 1816-1827.

Duffy, C. J. and Wurtz, R. H. (1991). Sensitivity of MST neurons to optic flow stimuli. I. A continuum of response selectivity to large-field stimuli, J. Neurophysiol. 65, 1329-1345.

Duffy, C. J. and Wurtz, R. H. (1995). Responses of monkey MST neurons to optic flow stimuli with shifted centers of motion, J. Neurosci. 15, 5192-5208. 
Dukelow, S. P., DeSouza, J. F. X., Culham, J. C., Van den Berg, A. V., Menon, R. S. and Vilis, T. (2001). Distinguishing subregions of the human MT+ complex using visual fields and pursuit eye movements, J. Neurophysiol. 86, 1991-2000.

Ernst, M. and Banks, M. (2002). Humans integrate visual and haptic information in a statistically optimal fashion, Nature 415, 429-433.

Fan, R. H., Liu, S., DeAngelis, G. C. and Angelaki, D. E. (2015). Heading tuning in macaque area V6, J. Neurosci. 35, 16303-16314.

Fasold, O., von Brevern, M., Kuhberg, M., Ploner, C. J., Villringer, A., Lempert, T. and Wenzel, R. (2002). Human vestibular cortex as identified with caloric stimulation in functional magnetic resonance imaging, NeuroImage 17, 1384-1393.

Fetsch, C. R., Wang, S., Gu, Y., DeAngelis, G. C. and Angelaki, D. E. (2007). Spatial reference frames of visual, vestibular, and multimodal heading signals in the dorsal subdivision of the medial superior temporal area, J. Neurosci. 27, 700-712.

Fetsch, C., Turner, A., DeAngelis, G. and Angelaki, D. (2009). Dynamic reweighting of visual and vestibular cues during self-motion perception, J. Neurosci. 29, 15601-15612.

Fetsch, C. R., Rajguru, S. M., Karunaratne, A., Gu, Y., Angelaki, D. E. and DeAngelis, G. C. (2010). Spatiotemporal properties of vestibular responses in area MSTd, J. Neurophysiol. 104, 1506-1522.

Fetsch, C. R., Pouget, A., DeAngelis, G. C. and Angelaki, D. E. (2012). Neural correlates of reliability-based cue weighting during multisensory integration, Nat. Neurosci. 15, 146154.

Fetsch, C. R., DeAngelis, G. C. and Angelaki, D. E. (2013). Bridging the gap between theories of sensory cue integration and the physiology of multisensory neurons, Nat. Rev. Neurosci. 14, 429-442.

Fitzpatrick, R. and Day, B. (2004). Probing the human vestibular system with galvanic stimulation, J. Appl. Physiol. 96, 2301-2316.

Frank, S. M., Baumann, O., Mattingley, J. B. and Greenlee, M. W. (2014). Vestibular and visual responses in human posterior insular cortex, J. Neurophysiol. 112, 2481-2491.

Frank, S. M., Wirth, A. M. and Greenlee, M. W. (2016a). Visual-vestibular processing in the human Sylvian fissure, J. Neurophysiol. 116, 263-271.

Frank, S. M., Sun, L., Forster, L., Tse, P. U. and Greenlee, M. W. (2016b). Cross-modal attention effects in the vestibular cortex during attentive tracking of moving objects, J. Neurosci. 36, 12720-12728.

Furlan, M., Wann, J. P. and Smith, A. T. (2014). A representation of changing heading direction in human cortical areas pVIP and CSv, Cereb. Cortex 24, 2848-2858.

Galletti, C., Battaglini, P. P. and Fattori, P. (1991). Functional properties of neurons in the anterior bank of the parieto-occipital sulcus of the macaque monkey, Eur. J. Neurosci. 3, 452-461.

Galletti, C., Fattori, P., Battaglini, P. P., Shipp, S. and Zeki, S. (1996). Functional demarcation of a border between areas $\mathrm{V} 6$ and V6A in the superior parietal gyrus of the macaque monkey, Eur. J. Neurosci. 8, 30-52.

Galletti, C., Fattori, P., Gamberini, M. and Kutz, D. F. (1999). The cortical visual area V6: brain location and visual topography, Eur. J. Neurosci. 11, 3922-3936.

Gernandt, B. (1949). Responses of mammalian vestibular neurons to horizontal rotation and caloric stimulation, J. Neurophysiol. 12, 173-184. 
Gibson, J. J. (1950). The Perception of the Visual World. Houghton Mifflin, Boston, MA, USA. Graziano, M., Andersen, R. and Snowden, R. (1994). Tuning of MST neurons to spiral motions, J. Neurosci. 14, 54-67.

Gu, Y., Watkins, P. V., Angelaki, D. E. and DeAngelis, G. C. (2006). Visual and nonvisual contributions to three-dimensional heading selectivity in the medial superior temporal area, J. Neurosci. 26, 73-85.

Gu, Y., DeAngelis, G. C. and Angelaki, D. E. (2007). A functional link between area MSTd and heading perception based on vestibular signals, Nat. Neurosci. 10, 1038-1047.

Gu, Y., Angelaki, D. E. and DeAngelis, G. C. (2008). Neural correlates of multisensory cue integration in macaque MSTd, Nat. Neurosci. 11, 1201-1210.

Gu, Y., Fetsch, C. R., Adeyemo, B., DeAngelis, G. C. and Angelaki, D. E. (2010). Decoding of MSTd population activity accounts for variations in the precision of heading perception, Neuron 66, 596-609.

Gu, Y., DeAngelis, G. C. and Angelaki, D. E. (2012). Causal links between dorsal medial superior temporal area neurons and multisensory heading perception, J. Neurosci. 32, 22992313.

Gu, Y., Cheng, Z., Yang, L., DeAngelis, G. C. and Angelaki, D. E. (2016). Multisensory convergence of visual and vestibular heading cues in the pursuit area of the frontal eye field, Cereb. Cortex 26, 3785-3801.

Guldin, W. and Grüsser, O.-J. (1998). Is there a vestibular cortex? Trends Neurosci. 21, 254 259.

Guldin, W. O., Akbarian, S. and Grüsser, O.-J. (1992). Cortico-cortical connections and cytoarchitectonics of the primate vestibular cortex: a study in squirrel monkeys (Saimiri sciureus), J. Comp. Neurol. 326, 375-401.

Haller, S., Fasler, D., Ohlendorf, S., Radue, E. W. and Greenlee, M. W. (2008). Neural activation associated with corrective saccades during tasks with fixation, pursuit and saccades, Exp. Brain Res. 184, 83-94.

Harris, L. (1994). Visual motion caused by movements of the eye, head and body, in: Visual Detection of Motion, A. T. Smith and R. J. Snowden (Eds). Academic Press, London, UK and San Diego, CA, USA.

Hikosaka, K., Iwai, E., Saito, H. and Tanaka, K. (1988). Polysensory properties of neurons in the anterior bank of the caudal superior temporal sulcus of the macaque monkey, J. Neurophysiol. 60, 1615-1637.

Huk, A. C., Dougherty, R. F. and Heeger, D. J. (2002). Retinotopy and functional subdivision of human areas MT and MST, J. Neurosci. 22, 7195-7205.

Kim, H., Pitkow, X., Angelaki, D. and DeAngelis, G. (2016). A simple approach to ignoring irrelevant variables by population decoding based on multisensory neurons, J. Neurophysiol. 116, 1449-1467.

Kimmig, H., Greenlee, M. W., Gondan, M., Schira, M., Kassubek, J. and Mergner, T. (2001). Relationship between saccadic eye movements and cortical activity as measured by fMRI: quantitative and qualitative aspects, Exp. Brain Res. 141, 184-194.

Kimmig, H., Ohlendorf, S., Speck, O., Sprenger, A., Rutschmann, R., Haller, S. and Greenlee, M. W. (2008). fMRI evidence for sensorimotor transformations in human cortex during smooth pursuit eye movements, Neuropsychologia 46, 2203-2213. 
Kirsch, V., Keeser, D., Hergenroeder, T., Erat, O., Ertl-Wagner, B., Brandt, T. and Dieterich, M. (2016). Structural and functional connectivity mapping of the vestibular circuitry from human brainstem to cortex, Brain Struct. Funct. 221, 1291-1308.

Knill, D. and Pouget, A. (2004). The Bayesian brain: the role of uncertainty in neural coding and computation, Trends Neurosci. 27, 712-719.

Kolster, H., Mandeville, J. B., Arsenault, J. T., Ekstrom, L. B., Wald, L. L. and Vanduffel, W. (2009). Visual field map clusters in macaque extrastriate visual cortex, J. Neurosci. 29, 7031-7039.

Kolster, H., Peeters, R. and Orban, G. A. (2010). The retinotopic organization of the human middle temporal area mt/v5 and its cortical neighbors, J. Neurosci. 30, 9801-9820.

Komatsu, H. and Wurtz, R. H. (1988). Relation of cortical areas MT and MST to pursuit eye movements. I. Localization and visual properties of neurons, J. Neurophysiol. 60, 580-603.

Koyama, M., Hasegawa, I., Osada, T., Adachi, Y., Nakahara, K. and Miyashita, Y. (2004). Functional magnetic resonance imaging of macaque monkeys performing visually guided saccade tasks: comparison of cortical eye fields with humans, Neuron 41, 795-807.

Lappe, M., Bremmer, F. and Van den Berg, A. V. (1999). Perception of self-motion from visual flow, Trends Cogn. Sci. 3, 329-336.

Large, I., Bridge, H., Ahmed, B., Clare, S., Kolasinski, J., Lam, W. W., Miller, K. L., Dyrby, T. B., Parker, A. J., Smith, J. E., Daubney, G., Sallet, J., Bell, A. H. and Krug, K. (2016). Individual differences in the alignment of structural and functional markers of the V5/MT complex in primates, Cereb. Cortex 26, 3928-3944.

Laurens, J., Liu, S., Yu, X.-J., Chan, R., Dickman, D., DeAngelis, G. C. and Angelaki, D. E. (2017). Transformation of spatiotemporal dynamics in the macaque vestibular system from otolith afferents to cortex, Elife 6, e20787. DOI:10.7554/eLife.20787.

Liu, S. and Angelaki, D. (2009). Vestibular signals in macaque extrastriate visual cortex are functionally appropriate for heading perception, J. Neurosci. 29, 8936-8945.

Lobel, E., Kleine, J., Le Bihan, D., Leroy-Willig, A. and Berthoz, A. (1998). Functional MRI of galvanic vestibular stimulation, J. Neurophysiol. 80, 2699-2709.

Lopez, C. and Blanke, O. (2011). The thalamocortical vestibular system in animals and humans, Brain Res. Rev. 67, 119-146.

Luna, B., Thulborn, K., Strojwas, M., McCurtain, B., Berman, R., Genovese, C. and Sweeney, J. (1998). Dorsal cortical regions subserving visually guided saccades in humans: an fMRI study, Cereb. Cortex 8, 40-47.

Mineault, P. J., Khawaja, F. A., Butts, D. A. and Pack, C. C. (2012). Hierarchical processing of complex motion along the primate dorsal visual pathway, Proc. Natl. Acad. Sci. USA 109, E972-E980.

Morgan, M. L., DeAngelis, G. C. and Angelaki, D. E. (2008). Multisensory integration in macaque visual cortex depends on cue reliability, Neuron 59, 662-673.

Ohshiro, T., Angelaki, D. E. and DeAngelis, G. C. (2011). A normalization model of multisensory integration, Nat. Neurosci. 14, 775-782.

Olson, C., Musil, S. and Goldberg, M. (1993). Posterior cingulate cortex and visuospatial cognition: properties of single neurons in the behaving monkey, in: Neurobiolgy of Cingulate Cortex and Limbic Thalamus: a Comprehesive Handbook, B. A. Vogt and M. Gabriels (Eds), pp. 366-380. Birkhauser, Boston, MA, USA. 
Orban, G. A., Claeys, K., Nelissen, K., Smans, R., Sunaert, S., Todd, J. T., Wardak, C., Durand, J. B. and Vanduffel, W. (2006). Mapping the parietal cortex of human and non-human primates, Neuropsychologia 44, 2647-2667.

Page, W. K. and Duffy, C. J. (1999). MST neuronal responses to heading direction during pursuit eye movements, J. Neurophysiol. 81, 596-610.

Page, W. and Duffy, C. (2003). Heading representation in MST: sensory interactions and population encoding, J. Neurophysiol. 89, 1994-2013.

Petit, L., Clark, V. P., Ingeholm, J. and Haxby, J. V. (1997). Dissociation of saccade-related and pursuit-related activation in human frontal eye fields as revealed by fMRI, J. Neurophysiol. 77, 3386-3390.

Pitzalis, S., Galletti, C., Huang, R.-S., Patria, F., Committeri, G., Galati, G., Fattori, P. and Sereno, M. I. (2006). Wide-field retinotopy defines human cortical visual area V6, J. Neurosci. 26, 7962-7963.

Pitzalis, S., Sereno, M. I., Committeri, G., Fattori, P., Galati, G., Patria, F. and Galletti, C. (2010). Human V6: the medial motion area, Cereb. Cortex 20, 411-424.

Pitzalis, S., Sdoia, S., Bultrini, A., Committeri, G., Di Russo, F., Fattori, P., Galletti, C. and Galati, G. (2013a). Selectivity to translational egomotion in human brain motion areas, PLoS One 8, e60241. DOI:10.1371/journal.pone.0060241.

Pitzalis, S., Fattori, P. and Galletti, C. (2013b). The functional role of the medial motion area V6, Front. Behav. Neurosci. 6, 91. DOI:10.3389/fnbeh.2012.00091.

Pitzalis, S., Sereno, M. I., Committeri, G., Fattori, P., Galati, G., Tosoni, A. and Galletti, C. (2013c). The human homologue of macaque area V6A, NeuroImage 82, 517-530.

Quinlan, D. J. and Culham, J. C. (2007). fMRI reveals a preference for near viewing in the human parieto-occipital cortex, NeuroImage 36, 167-187.

Roberts, R. E., Ahmad, H., Arshad, Q., Patel, M., Dima, D., Leech, R., Seemungal, B. M., Sharp, D. J. and Bronstein, A. M. (in press). Functional neuroimaging of visuo-vestibular interaction, Brain Struct. Funct. DOI:10.1007/s00429-016-1344-4.

Royden, C. and Hildreth, E. (1996). Human heading judgments in the presence of moving objects, Percept. Psychophys. 58, 836-856.

Saito, H.-A., Yukie, M., Tanaka, K., Hikosaka, K., Fukada, Y. and Iwai, E. (1986). Integration of direction signals of image motion in the superior temporal sulcus of the macaque monkey, J. Neurosci. 6, 145-157.

Schaafsma, S. J. and Duysens, J. (1996). Neurons in the ventral intraparietal area of awake macaque monkey closely resemble neurons in the dorsal part of the medial superior temporal area in their responses to optic flow patterns, J. Neurophysiol. 76, 4056-4068.

Schlack, A., Hoffmann, K. P. and Bremmer, F. (2002). Interaction of linear vestibular and visual stimulation in the macaque ventral intraparietal area (VIP), Eur. J. Neurosci. 16, 1877-1886.

Schlack, A., Sterbing-D’Angelo, S. J., Hartung, K., Hoffmann, K.-P. and Bremmer, F. (2005). Multisensory space representations in the macaque ventral intraparietal area, J. Neurosci. 25, 4616-4625.

Smith, A. T., Wall, M. B. and Thilo, K. V. (2012). Vestibular inputs to human motion-sensitive visual cortex, Cereb. Cortex 22, 1068-1077.

Smith, A. T., Beer, A. L., Furlan, M. and Mars, R. B. (in press). Connectivity of the cingulate sulcus visual area (CSv) in the human cerebral cortex, Cereb. Cortex. DOI:10.1093/cercor/ bhx002. 
Stephan, T., Deutschländer, A., Nolte, A., Schneider, E., Wiesmann, M., Brandt, T. and Dieterich, M. (2005). Functional MRI of galvanic vestibular stimulation with alternating currents at different frequencies, NeuroImage 26, 721-732.

Sunaert, S., VanHecke, P., Marchal, G. and Orban, G. A. (1999). Motion-responsive regions of the human brain, Exp. Brain Res. 127, 355-370.

Sunkara, A., DeAngelis, G. C. and Angelaki, D. E. (2016). Joint representation of translational and rotational components of optic flow in parietal cortex, Proc. Natl. Acad. Sci. USA 113, 5077-5082.

Suzuki, M., Kitano, H., Ito, R., Kitanishi, T., Yazawa, Y., Ogawa, T., Shiino, A. and Kitajima, K. (2001). Cortical and subcortical vestibular response to caloric stimulation detected by functional magnetic resonance imaging, Cogn. Brain Res. 12, 441-449.

Swisher, J. D., Halko, M. A., Merabet, L. B., McMains, S. A. and Somers, D. C. (2007). Visual topography of human intraparietal sulcus, J. Neurosci. 27, 5326-5337.

Takahashi, K., Gu, Y., May, P. J., Newlands, S. D., DeAngelis, G. C. and Angelaki, D. E. (2007). Multimodal coding of three-dimensional rotation and translation in area MSTd: comparison of visual and vestibular selectivity, J. Neurosci. 27, 9742-9768.

Tanabe, J., Tregellas, J., Miller, D., Ross, R. G. and Freedman, R. (2002). Brain activation during smooth-pursuit eye movements, NeuroImage 17, 1315-1324.

Tanaka, K. and Saito, H. (1989). Analysis of motion of the visual field by direction, expansion/contraction, and rotation cells clustered in the dorsal part of the medial superior temporal area of the macaque monkey, J. Neurophysiol. 62, 626-641.

Tanaka, K., Fukada, Y. and Saito, H. (1989). Underlying mechanisms of the reponse specificity of the expansion/contraction and rotation cells in the dorsal part of the medial superior temporal area of the macaque monkey, J. Neurophysiol. 62, 642-656.

Telford, L., Howard, I. P. and Ohmi, M. (1995). Heading judgments during active and passive self-motion, Exp. Brain Res. 104, 502-510.

Van Essen, D., Drury, H., Dickson, J., Harwell, J., Hanlon, D. and Anderson, C. (2001). An integrated software suite for surface-based analysis of visual cortex, J. Am. Med. Inform. Assoc. 8, 443-459.

von Holst, E. and Mittelstaedt, H. (1950). Das Reafferenzprinzip, Naturwissenschaften 37, 464 476.

Wall, M. B. and Smith, A. T. (2008). The representation of egomotion in the human brain, Curr. Biol. 18, 191-194.

Wall, M. B., Lingnau, A., Ashida, H. and Smith, A. T. (2008). Selective visual responses to expansion and rotation in the human MT complex revealed by functional magnetic resonance imaging adaptation, Eur. J. Neurosci. 27, 2747-2757.

Warren, W. H. and Hannon, D. J. (1988). Direction of self-motion is perceived from optical flow, Nature 336, 162-163.

Warren, P. A. and Rushton, S. K. (2009). Optic flow processing for the assessment of object movement during ego movement, Curr. Biol. 19, 1555-1560.

Wirth, A. M., Frank, S. M., Beer, A. L. and Greenlee, M. W. (subm.). White matter connections of the visual-vestibular cortex.

Yang, Y., Liu, S., Chowdhury, S. A., DeAngelis, G. C. and Angelaki, D. E. (2011). Binocular disparity tuning and visual-vestibular congruency of multisensory neurons in macaque parietal cortex, J. Neurosci. 31, 17905-17916. 
Zhang, T. and Britten, K. H. (2010). The responses of VIP neurons are sufficiently sensitive to support heading judgments, J. Neurophysiol. 103, 1865-1873.

Zhang, T., Heuer, H. W. and Britten, K. H. (2004). Parietal area VIP neuronal responses to heading stimuli are encoded in head-centered coordinates, Neuron 42, 993-1001. 